\title{
EFFICIENCY OF PRODUCTION OF X RAYS
}

\author{
By Warren W. Nicholas
}

\section{ABSTRACT}

Efficiency.-The various corrections necessary for an estimate of efficiency of production $E_{c}$ of continuous spectrum $\mathrm{X}$ rays are carefully considered. These include corrections for absorption (including absorption in the anticathode), reflection of cathode rays at the focal spot, asymmetric spatial distribution of $\mathrm{X}$-ray intensity, and allowance for characteristic ray energy. Applying these corrections to some data by Rump, $E_{c}$ for a tungsten anticathode at a voltage $V=40 \mathrm{kv}$ is found to be 0.0039 . It is pointed out that $E_{c}$ can not yet be accurately estimated for a wide range of voltage. Present knowledge of secondary processes in gas filled tubes is so limited as to make it inadvisable to attempt to use these tubes for an exact estimate of $E_{c}$. The bearing of the Thomson-Whiddington constant on the generation of X-ray energy is pointed out, and an explanation given for Ulrey's results indicating a certain degree of periodicity in the dependence of efficiency on atomic number.

Intensities. - It is shown, on the basis of Rump's data, that the continuous spectrum intensity generated inside an anticathode and radiated at an angle $\psi=90^{\circ}$ with the cathode stream is proportional to $V^{3 / 2}$, at least for tungsten and $V>40 \mathrm{kv}$. A general formula for the spectral intensity distribution of this radiation is given, as well as a means for estimating its variation with $\psi$; absorption in the anticathode is described quantitatively; the proportion of $K$ characteristic rays for tungsten is found. There is thus obtained a completa description of the intensities obtainable outside X-ray tubes for a wide range of practical conditions.

CONTENTS

Page

843

845

845

845

847

851

853

857

859

861

863

864.

865

\section{INTRODUCTION}

During some recent work for the International Critical Tables and for the Smithsonian Physical Tables, the author had occasion to review critically the experimental work that has been done on the measurement of the total energy in the X-ray continuous spectrum. There is a surprising range of values obtained in this work. Especially, the recent measurement by Rump (1) ${ }^{1}$, who used what seems

1 The figures given in parentheses here and throughout the text relate to the list of references given at the end of the paper.

$11295^{\circ}-30-5$ 
to be the best method for measuring $\mathrm{X}$-ray energy, is almost 50 per cent higher than the maximum value estimated by Kulenkampff (2) from a careful consideration of all previous work with unresolved energy. A closer approximation than this is very desirable if for no other reason than to check theoretical estimates of continuous spectrum energy (3). But also the $\mathrm{X}$-ray continuous spectrum is often a very convenient standard source of X-ray energy; for example, it has been used recently by Webster (4) for an estimate of the absolute probability of $K$ emission due to excitation by cathode rays. Further, it is only recently that a fairly complete conception has been obtained of the variation of X-ray intensity in different directions relative to the cathode stream (5). Inasmuch as this variation must be known in order to calculate the total energy emitted (which involves an integration over the whole sphere), it is of interest to apply the new results in an attempt to obtain a more satisfactory estimate of efficiency than it has been possible to obtain previously.

For purposes of definition, let a hypothetical X-ray tube be imagined, the electrodes of which are maintained at a constant d.c. potential difference $V$, and the current $i$ through which is all carried by the cathode rays. Suppose these cathode rays all enter the target at the focal spot without "reflection"; this insures that all the energy of the cathode rays is converted, in the focal spot, either into heat energy or X-ray energy; that is

$$
V i=H+J
$$

where $H$ is the heat energy, and $J$ the $\mathrm{X}$-ray energy, generated per second by the cathode rays within the focal spot in this hypothetical tube.

The quantity $J$ may be divided into several parts. One of these parts, $J_{c}$, is associated with the energy of the continuous spectrum $\mathrm{X}$ rays, and the remaining parts, $J_{K}, J_{L}, .$. . , are associated with the various groups of characteristic rays $K, L$, . . . , respectively. Thus

$$
J=J_{c}+J_{K}+J_{L}+\text {. . . }
$$

By efficiency of production of $\mathrm{X}$ rays is here understood

$$
E=J / V i
$$

by efficiency of production of continuous spectrum $\mathrm{X}$ rays

$$
E_{c}=J_{c} / V i
$$

and similarly, the efficiency of production of the various groups of characteristic rays, $K, L, \ldots$. , may be defined as

$$
E_{K}=J_{K} / V i, E_{L}=J_{L} / V i, \quad . \quad \text {. , }
$$

respectively. The present paper will deal particularly with an estimation, from experiment, of $E_{c}$ for a tungsten anticathode, although some data associated with $E_{K}$ will be obtained incidentally. 


\section{EXPERIMENTAL DIFFICULTIES}

\section{LOSSES DUE TO STRAY CATHODE RAYS}

In practice, the simple conditions outlined above in connection with the "hypothetical" tube do not hold. With the actual X-ray tube, the cathode rays do not convert all their energy into heat and $X$ rays at the focal spot, but due to a "reflection" (either a single large-angle deflection resulting from a close collision of a cathode ray with an atomic nucleus, or a succession of smaller deflections (6) many of the cathode rays leave the focal spot with a considerable fraction of their initial energy $(7,8,9,10)$. These "stray" rays may strike other parts of the tube (the walls, or parts of the anticathode remote from the focal spot) to generate "off-focus" X rays and heat. On this account, in the actual tube, the $\mathrm{X}$ ray and heat energies generated per second at the focal spot are not $J$ and $H$, but some quantities, say $J^{\prime}$ and $H^{\prime}$, where

$$
J^{\prime}<J \text { and } \mathrm{H}^{\prime}<H
$$

(Quantities associated only with the focal spot in the actual X-ray tube will be singly primed.) Similar considerations hold for the quantities $J^{\prime}{ }_{c}, J^{\prime}{ }_{k}$, etc., definitions of which are obvious from the above. It may be pointed out that $J-J^{\prime}$ and $H-H^{\prime}$ are not measures of the off-focus X ray and heat energies except for the particular case where all the stray cathode rays dissipate their energy in a material in which the efficiency of X-ray production is the same as for the material of the anticathode. The difference between $J$ and $J^{\prime}$ is not at all negligible, this difference, with a tungsten anticathode, and voltages of the order of $100 \mathrm{kv}$, being about 20 per cent of $J(7,8)$.

\section{SPECIAL DIFFICULTIES WITH GAS-FILLED TUBES}

In gas-filled tubes, some further complicating factors enter; it is desirable to discuss these factors at some length on account of the fact that so many estimates of efficiency have been based on work with this type of tube, and any possibility of comparison of these estimates with the present one should not be overlooked. The considerations discussed here are quite apart from the well-known difficulties of operation of gas-filled tubes, and deal rather with some ambiguities connected with the interpretation of data obtained with these tubes.

With gas-filled tubes, the current $i^{\prime \prime}$ (doubly primed quantities will be associated with phenomena in gas-filled tubes) read on a milliammeter in the tube circuit is not equal to the cathode ray current $i$; this is on account of the fact that a very appreciable part of the tube current $i^{\prime \prime}$ is carried by positive ions. In the best work with these tubes, the current $i$ is determined by measuring the amount of heat $H^{\prime \prime}$ generated in the anticathode. The true cathode ray current $i$ is then assumed to be $H^{\prime \prime} / V$, and the efficiency $E^{\prime \prime}$ is taken to be $J^{\prime \prime} / H^{\prime \prime}$. It remains to ascertain how closely this approximates the true efficiency $E=J / V i=$ (approx.) $J / H$ (since $J$ is very small compared with $H$ ).

The difficulties here are again due to the reflection of cathode rays from the target surface, but there is a special uncertainty with gas- 
filled tubes on account of not knowing where the stray $\mathrm{X}$ rays are produced; for example, if they were produced only on the anode stem, as in a Coolidge tube, $H^{\prime \prime}$ would include the heat energy produced by the stray cathode rays. However, as is well known, the reason the cathode rays in a well-evacuated tube (Coolidge tube) must eventually dissipate their energy in the anode is that the inner walls of the glass acquire a negative charge due to the accumulation of stray cathode rays, and since the leakage is small, the walls quickly acquire such a potential as will prevent additional stray cathode rays striking them; this is evidenced by the absence of fluorescence on the walls of the Coolidge tube. But in a gas tube the walls do not become charged to the extent they do in the Coolidge tube; this is shown by the fact that they continually exhibit a bright fluorescence. Thus it may be expected that, in a gas tube, an appreciable portion of the cathode rays strike the glass walls with high enough speeds to gencrate considerable X-ray energy and heat energy there.

Now the effect of these conditions on the comparison of $E$ with $E^{\prime \prime}$ may be illustrated by some special cases. Suppose first that 20 per cent, say, of the cathode rays are reflected from the target face with their entire energy, and that they all go to the glass walls and stay there. Then it is evident that $J^{\prime \prime}$ and $H^{\prime \prime}$ will for this case be 80 per cent of $J$ and $H$, respectively, and that $E^{\prime \prime}$, will be equal to $E$. On the other hand, suppose the reflected cathode rays lose only a negligible amount of energy in the glass walls; and that, due to the field between anode and walls, they are drawn back to the anode, striking it at such points as to contribute to $H^{\prime \prime}$ but not to $J^{\prime \prime}$. In this case $E^{\prime \prime}$ will obviously be 80 per cent of $E$. For the other extreme suppose that a fraction $a$ of the cathode rays first lose a portion $\beta$ of their energy in the focal spot, then reflect to the glass walls and stay there. Had these rays remained in the focal spot, they would have generated there X-ray energy per second $\Delta J=J-J^{\prime \prime}$ and heat energy $\Delta H=H-H^{\prime \prime}$ with an efficiency $\Delta J / \Delta H$. This efficiency would have been less than the true efficiency $J / H$, since efficiency decreases with decreasing cathode ray speed. Thus the loss to the anticathode of these slow-speed stray cathode rays increases the apparent efficiency (that is, $E^{\prime \prime}=\frac{J-\Delta J}{H-\Delta H}>\frac{J}{H}$, as may be easily shown).

For example, if $a$ were 0.3 and $\beta 0.2, E^{\prime \prime}$ would be about 6 per cent greater than $E$.

The actual processes occurring in gas-filled tubes are probably a combination of the above. Cathode rays reflect from the focal spot with various portions of their original speeds, and partially charge the walls of the tube. This allows only the faster rays to strike the walls; the slower ones are pulled back to the anode, striking, in general, at points other than the focal spot. So far as the author is aware, no data is available for a quantitative estimate of these processes, and, therefore, an estimate of the true efficiency $E$ from the apparent -fficiency $E^{\prime \prime}$ ubtained with gas filled tubes can not be relied upon to better than about 10 or 20 per cent of its value. Moreorer, it would seem very difficult to ever make a satisfactory estimate of the many experimental errors introduced by these complications. In a Coolidge 
tube, on the other hand, the possibility of making satisfactory ('orrections for the errors introduced by the reflection of cathode rays from the focal spot seems much less remote. For in this case all the reflected rays must eventually return to the anode without loss of speed and the resulting $\mathrm{X}$-ray energy generated there can be estimated. Further, as mentioned above, the tube current as measured with a milliammeter is, with the hot filament tube, presumably a quantity which can be interpreted without ambiguity. For these reasons, it seems preferable to base the present estimate of efficiency ertirely on work done with Coolidge tubes; specifically, the work by Rump (1) was chosen as being apparently the most satisfactory from the standpoint of the measurement of the X-ray energy, for the use of fairly steady voltages, and for the use of a range of voltage (40 to $150 \mathrm{kv}$ ) which is of especial practical interest at present.

\section{LOSSES DUE TO ABSORPTION}

$J$ and $J^{\prime}$ are associated, according to definition, with X-rny encrgy produced inside the anticathode; thus they can not be measured directly, but must be calculated from quantities which are directly measurable. In measurements of X-ray efficiency the quantity measured is an X-ray intensity $g I^{\prime}$, the unresolved energy per second incident on some definite area $A$ outside the tube. The slit system limiting the beam to $A$ usually also limits the registered intensity to rays which come from the focal spot; hence $g I^{\prime}$ must be associated with $J^{\prime}$ instead of $J$. Now the rays reaching $A$ must have passed through several materials and lost thereto an appreciable fraction of their initial energy. If $\mathrm{I}^{\prime}$ is defined as the unresolved intensity which would have been registered at $A$ had these various absorptions not occurred, then $g$ represents that fraction of the initial energy which remains after the various absorptions have taken place. From present knowledge of absorption coefficients, the calculation of that part of $g$ due to the walls of the tube, air path, etc., is perfectly straightforward when the special energy distribution of $g I^{\prime}$ is known. The calculation of the part of $g$ due to absorption in the anticathode is not so simple, however, since for an adequate estimate a knowledge is required of the depths within the anticathode at which the various wave lengths are produced. It should be possible to calculate the depths indirectly from data on cathode rays involving their losses of speed and deflections on entering the anticathode, together with known laws of X-ray emission. The theoretical difficulties in such a calculation are, however, very great, and such data on cathode rays is not yet complete enough for general use. There is some direct experimental data on the depths in the anticathode at which $\mathrm{X}$ rays are produced, but the data dealing with spectrally resolved rays is meager, and most of it is open to some doubt on account of polarization considerations (9) and on account of complications introduced by the roughness of the target face. In view of the fact that the correction for absorption in the anticathode is the one correction in measurements of X-ray efficiency which can not be readily evaluated, it becomes of prime importance to obtain for quantitative estimates of efficiency a satisfactory expression for this correction. 
The formula used in the present work for the correction for absorption in the anticathode is the following:

$$
\bar{x}=k \frac{V}{b Z^{\frac{1}{2}}}\left\{1-e^{-\frac{V Z^{\frac{x}{2}}}{k}}\left(\frac{\nu_{0}-\nu}{\nu_{0}}\right)\right\}
$$

where $\bar{x}$ is the effective depth inside a target of atomic number $Z$ at which frequency $\nu$ in the continuous spectrum is produced, $\bar{x}$ being measured in the direction in which the cathode beam strikes the target face. $V$ is the voltage applied to the tube, which also determines the frequency $\nu_{0}$ of the quantum limit, $b$ is the constant of the Thomson-Whiddington-Bohr law in the form

$$
\frac{d\left(V^{2}\right)}{d x}=-b
$$

$e$ is the base of natural logarithms, and $k$ is a constant probably dependent on the angle at which the cathode rays strike the target face. For the present case this angle was $45^{\circ}$ to a close enough approximation; ${ }^{2}$ and since the $\mathrm{X}$ rays were taken off in the usual direction, the effective path length the $\mathrm{X}$ rays must traverse in the anticathode is also $\bar{x}$. $V$ is expressed in kilovolts; $\bar{x}$ is in centimeters; $b$ is calculated from Terrill's formula $b=40 \times 10^{4} \rho$. where $\rho$ is the density (in $\mathrm{g} \cdot \mathrm{cm}^{-3}$ ) of the anticathode material. The numerical value of $k$ was taken to be 120 ; this seemed to agree quite satisfactorily with the empirical data, as will be shown below.

The derivation of the formula was based on the following considerations: If the cathode rays penetrated the anticathode without being deflected (or "diffused"), the depth $p$ (measured along the line of the cathode ray beam) to which they would penetrate would be given by the Thomson-Whiddington-Bohr law $p=\frac{V^{2}}{b}$. Other things being equal, this law will be a measure, for different anticathodes, of the depths to which the cathode rays penetrate in being reduced to zero speed. Thus it may be supposed that the mean depth $p$ to which the cathode rays actually penetrate (that is, now taking account of deflections) will be given by a law of the form

$$
p=\frac{V^{2}}{b} f(Z, V)
$$

where $f(Z, V)$ is a factor designed to reduce $p$ to a fraction of $p$ which is dependent on the extent of the deflections of the cathode rays. It should be noted now that the greater the total path length of the cathode rays in the material the more numerous the deflections along the whole path. Accordingly, for very short paths (that is, for low initial speeds), the rays may be expected to be little deflected, and reach depths approximating $p$, whereas for long paths the fraction $\bar{p} / p$ may be expected to become smaller, since the total number of deflections along the path has increased. Thus a form for $f(Z, V)$ is required which will become unity for very short path lengths, and for long paths will gradually approach some other function $j(Z, V)$

2 Actually, with the customary arrangement of eleetrodes, this angle is somewhat smaller than $45^{\circ}$ (meas-

2 Actually, with the customary arranger, face for, as was pointed out by Webster and Hennings (11), the
ured from the normal to the target faces an 
which is a measure of the influence of deflections on penetration for the higher voltages. A form for $f(Z, V)$ which fulfills these condi-
tions is

$$
f=j\left\{1-e^{-1 / j}\right\}
$$

( $j$ is large for short paths, and small for long paths, see below). Substituting in (4) we have for the mean depth of penetration

Although the form

$$
\bar{p}=\frac{V^{2}}{b} j(Z, V)\left\{1-e^{-1 / j(Z, V)}\right\}
$$

$$
j=C / V Z^{\frac{1}{2}}
$$

was chosen to fit the X-ray data, it is interesting that, choosing the constant $C=35 \mathrm{kv}$, this formula gives the order of magnitude of some data by Schonland (12) on cathode ray penetration, and for a very large range of atomic number and voltage. From Schonland's data may be obtained the thickness of metal foil which "reflects" as many cathode rays of a given voltage as it transmits. Half this thickness was taken to be the mean depth of penetration, for if the cathode rays are completely diffused (that is, have random directions) by the time they reach this mean depth, then they have equal probabilities of reaching the two surfaces of the foil. Considering the rough character of this approximation, the comparison with Schonland's data in

\begin{tabular}{|c|c|c|c|c|}
\hline$Z$ & $V$ & $\vec{p}$ (calc) & $\bar{p}$ (obs) & Ratio \\
\hline $13(\mathrm{Al})$ & $\begin{array}{l}k v \\
87 \\
41 \\
24 \\
20 \\
14\end{array}$ & $\begin{array}{c}\mu \\
7.8 \\
3.6 \\
2.0 \\
.10 \\
.97\end{array}$ & $\begin{array}{c}\mu \\
11.0 \\
3.5 \\
1.3 \\
.09 \\
.38\end{array}$ & $\begin{array}{l}1.4 \\
1.0 \\
.64 \\
.9 \\
.4\end{array}$ \\
\hline
\end{tabular}
Table 1 may be regarded as satisfactory.

TABLE 1

Finally, the formula for $\bar{p}$ must be modified in such a manner as to describe the mean depth $\bar{x}$ of production of the $\mathrm{X}$ rays, which will depend on frequency $\nu$ as well as on $V$ and $Z$. First it may be pointed out that the rays of very low frequency are produced by the cathode rays all along their paths, according to well-known laws of X-ray emission $(13,9)$. Thus, $\bar{p}$ may be regarded as a measure of $\bar{x}_{\nu=0}$ except that the latter quantity will be uniformly smaller than $\bar{p}$. This condition was expressed by assuming that for low frequencies the formula for $\bar{x}$ must approximate

$$
k \frac{V}{b Z^{1 / 2}}\left\{1-e^{-V Z^{3 / 2} l_{k}}\right\}
$$

which is the same as the formula for $\bar{p}$ except for a different numerical value for the constant (the value adopted for $k$ was such as to make $\bar{p} / \bar{x}_{\nu=0}$ about 3 for ordinary conditions, which is reasonable. The higher frequencies, however, must be produced at shallower depths, since it is only in that part of their path for which the cathode rays retain a large fraction of their original speed (and consequently have not penetrated so deeply) that they are allowed by quantum laws to 
produce the higher frequencies. For example, a frequency $\nu_{x}$ corresponding to a voltage $V_{x}$ which is near $V$, the voltage on the tube, can not be produced at a depth greater than

$$
x=\frac{V^{2}-V_{x}^{2}}{b}
$$

which is the depth to which a cathode ray can penetrate, according to the Thomson-Whiddington-Bohr law and retain a speed sufficient, by quantum laws, to enable it to produce $\mathrm{X}$ rays of frequency $\nu_{x}$. When $V-V_{x}$ is small, the cathode rays will not have been much deflected before reaching depth $x$; also, according to the laws of emission, they will have produced $\mathrm{X}$ rays of frequency $\nu_{x}$ with roughly uniform intensity at all depths between zero and $x$. Thus for this special case, as has been pointed out by Webster and Hennings (11), the mean depth approximates half of $x$, or

$$
\frac{V^{2}-V_{x}^{2}}{2 b}
$$

and since $\left(V-V_{\mathrm{x}}\right) \ll V$ for the case considered, (9) is approximated by

$$
\frac{V\left(V-V_{\mathrm{x}}\right)}{b}
$$

Thus the final formula for $\bar{x}$ should approximate (8) for low frequencies and (10) for high frequencies. The formula (2) adopted does this, since

and

$$
1-e^{-x} \rightarrow x \text { for } x \rightarrow 0
$$

$$
\frac{\nu_{0}-\nu}{\nu_{0}}=\frac{V-V_{x}}{V}
$$

and gives a reasonable distribution of depths for the intermediate frequencies. Some numerical comparisons with experimental results are given in Table 2. The best data for the comparison is that of Webster and Hennings; the calculated values agree excellently for the higher voltages. The disagreement at the lower voltages is believed to be due to a difficulty with the experimental method, since the experimental values for mean depth are greater than the maximum depths allowed by the Thomson-Whiddington-Bohr law if the constant $b$ is calculated according to Terrill's formula. And although $b$ has not yet been obtained directly for molybdenum, there is reason to believe that this formula will apply with good approximation; these questions will be discussed in detail below. The other data, with the exception of the author's, are for unresolved radiation, and are probably complicated by the presence of characteristic rays. On account of fluorescent excitation, these rays have a much greater mean depth of production than the continuous radiation (14); for comparison with this data the value zero was used for $\nu$ in formula (2). 
However, there seems to be no reason at present, either experimental or theoretical, to doubt this extrapolation.

Knowing the spectral intensity distributions for radiation generated inside the anticathode, the distributions obtained outside the anticathode are readily obtained by use of the above formula for effective depth of production, together with known absorption coefficients. The formulas below, which are equivalent to that given by Richtmyer, were used for the latter, for wave lengths shorter than the $L$ critical absorption limits:

$$
\begin{aligned}
& \mu=\frac{\rho}{A}\left(0.0136 Z^{4} \lambda^{3}+0.32 Z\right) \ldots \lambda<\lambda_{K} \\
& \mu=\frac{\rho}{A}\left(0.0020 Z^{4} \lambda^{3}+0.32 Z\right) \ldots \lambda_{K}<\lambda<\lambda_{L_{I}}
\end{aligned}
$$

where $\mu$ is the absorption coefficient defined in the usual manner, $\rho$ is the density, $Z$ the atomic number, and $A$ the atomic weight of

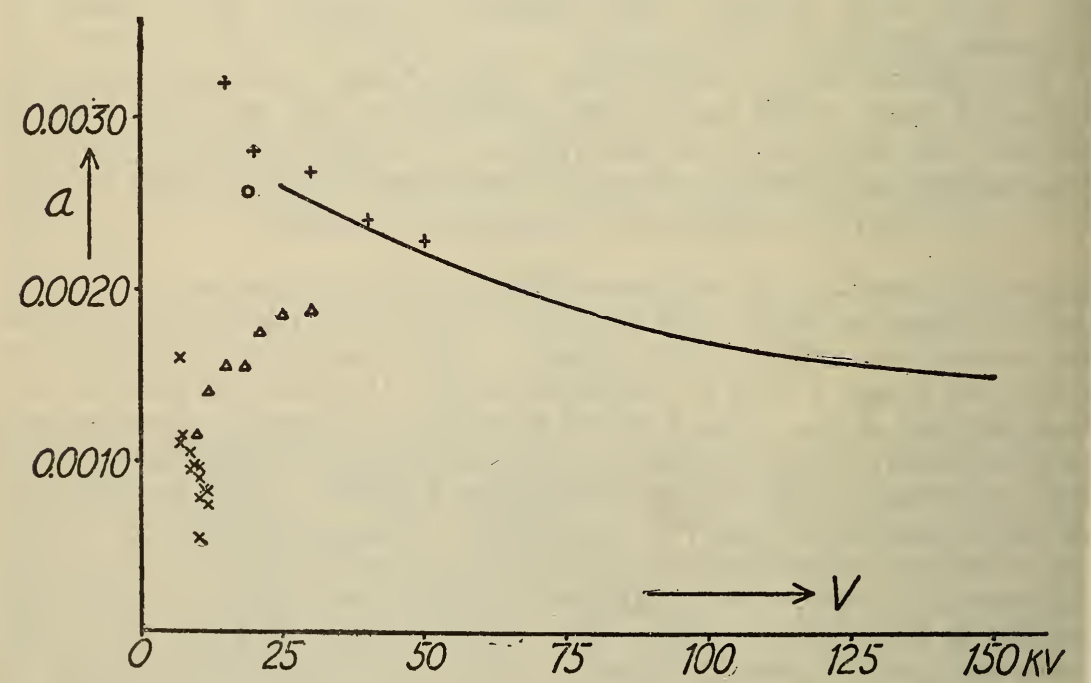

FIgURE 1.-Values for the quantity a used in the formula for spectral intensity distribution

$\times$ Observations of Kulenkampff (18) for a range of atomic number (Z) from 13 to 78.

$\triangle$ Webster and Hennings $(13)$ on molybdenum $(\eta=42)$

+ Nicholas $(9)$ on copper $(Z=29)$.

the absorbing material. The upper formula applies to wave lengths ( $\lambda$ ) shorter than the $K$ limit $\left(\lambda_{K}\right)$, and the lower to the region between the $K$ and the $L$. For wave lengths longer than $\lambda_{L_{I}}$, which are really of no importance in the present esimate, the $\lambda^{3}$ formula was used in conjunction with some data by Allen taken from Compton's " $\mathrm{X}$ rays and Electrons." Also the spectral intensity distribution for any subsequent filtration is now readily calculated. The distribution (curve C) of Figure 2 is the spectrum obtained by Rump in his calorimeter according to present calculations; the filtration in tube walls, air path, calorimeter walls, etc., was closely equivalent to $1.23 \mathrm{~mm}$ aluminum according to data given by Rump in his paper. The ratio 
(area under curve $C$ )/(area under curve $A$ ) of Figure 2 is the factor $g$ above in case the measured radiation consists entirely of continuous spectrum rays. In the same manner, spectra can be drawn for different tube voltages, the corrections for absorption carried through in the same way, and the value of $g$ obtained as a function of the voltage applied to the tube; the continuous curve of Figure 3 represents this dependence of $g$ on voltage. It should be noted that $g$ is simply a ratio of intensities, and thus is independent of the actual amount of energy produced or measured.

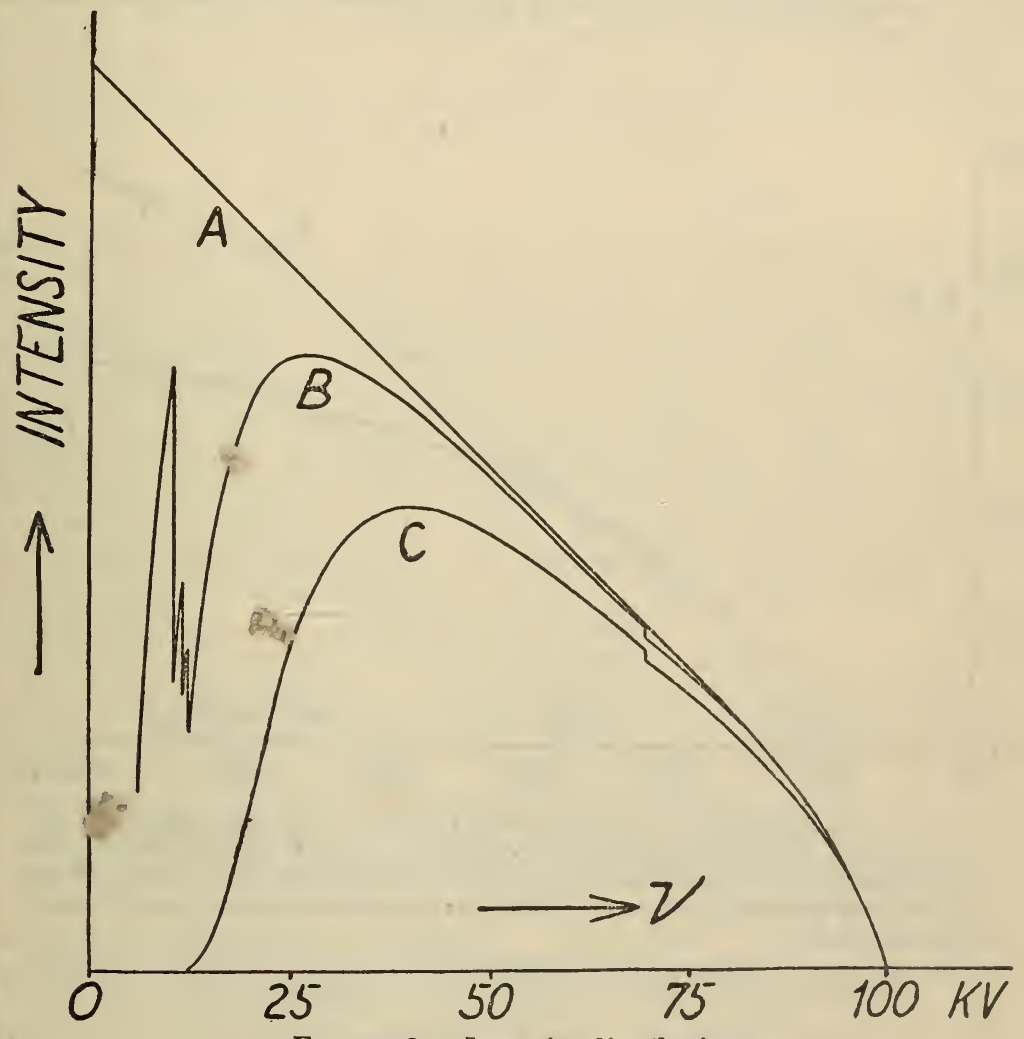

Figure 2.-Intensity distribution

$A$, spectral intensity distribution in the continuous radiation from tungsten at $100 \mathrm{kv}$, as produced within the anticathode, according to formula (11). Plotted on a frequency seale, with frequencies expressed in equivalent excitation potentials.

$B$, intensities outside the anticathode.

$C$, same after passing through tube walls, etc., equivalent to $1.23 \mathrm{~mm}$ aluminum.

The factor $g$ of Figure 3 (full line) is the ratio (area under $C$ )/(area under $A$ ).

\section{VARIATION OF X-RAY INTENSITY WITH VOLTAGE}

Experimentally, Rump measured an amount of energy corresponding to $g I^{\prime}$ and its variation with voltage. Knowing $g$ we can thus calculate $I^{\prime}$ from Rump's results and determine its variation with voltage $(V)$. It is usually accepted from the work at relatively high voltages that $I^{\prime}{ }_{c}$ is closely proportional to $V^{2}$ in the high voltage region, but in none of this work have corrections for absorption been so carefully considered as at present. A neglect of absorption will 
lead, in general, to too large an apparent value of the exponent, so it will not be surprising to find in the present work a somewhat less rapid increase of $I^{\prime}{ }_{c}$ with voltage. At low voltages, Kulenkampff has found that $I^{\prime}{ }_{c}$ increases somewhat less rapidly than $V^{2}$, as an integration of his formula will show. At high voltages his formula would lead to a variation of $I^{\prime}$ c approaching proportionality with $V^{2}$, but it has been shown (9) that this formula can not be extended to higher voltages without modification. In the present work, the calculations of $I^{\prime}{ }_{c}$ based on Rump's data and the present values of $g$ lead to a variation of $I^{\prime}{ }_{c}$ which is much more nearly proportional to $V^{3 / 2}$ than to $V^{2}$

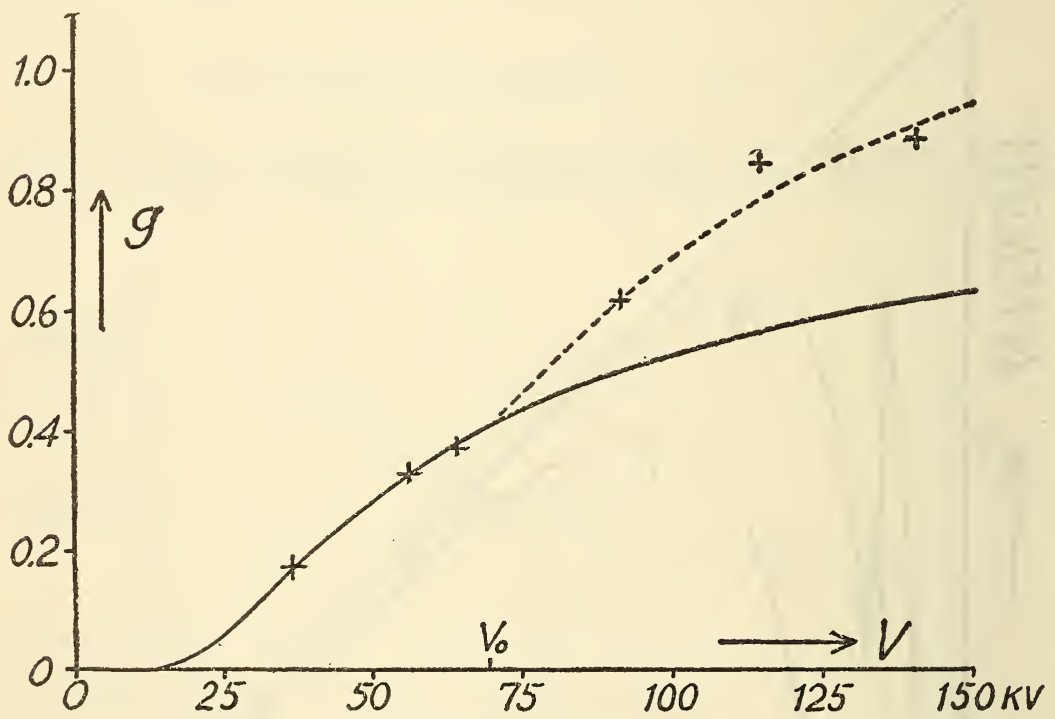

FigURe 3.-Comparison of Rump's data (crosses) with calculations which assume tctal continuous spectrum intensity produced inside the tungsten anticathode to be proportional not to $V^{2}$ (as has usually been assumed) but to $V^{3 / 2}$, and allowing for a considerable proportion of energy in the $K$ characteristic rays

The unbroken curve corresponds to the continuous radiation (see fig. 2) and the broken curve represents a correction for characteristic rays. Compare with Figure 4.

$$
I^{\prime}{ }_{c}=K V^{3 / 2} \ldots(40 \mathrm{kv}<V<150 \mathrm{kv})
$$

It should be stressed that it can not be concluded from the present work that this formula has general validity; indeed the indications are that the formula predicts too much energy for the region of $10 \mathrm{kv}$. However, there can be little doubt that, for a tungsten anticathode and for voltages between 40 and $150 \mathrm{kv}$ (and these are conditions of comparatively great practical importance), the formula closely approximates actual conditions. The numerical value adopted for $K$ was 0.043 where $V$ is in kilovolts and $I^{\prime}{ }_{c}$ is in ergs/sec. $\mathrm{cm}^{2}$ at a distance of $1 \mathrm{~m}$ from the focal spot, for 1 milliampere tube current.

From formula (12) for $I^{\prime}{ }_{c}$ and from Rump's experimentally measured values for $g I^{\prime}$ may be obtained values for the quantity $g$; these values are plotted in Figure 3 . The observed points agree well with the predicted curve below $69 \mathrm{kv}$, at which voltage the characteristic 
rays are produced. Above $69 \mathrm{kv}$ the difference between the observed and calculated values may be regarded as a measure of the energy contained in the $K$ characteristic rays of tungsten. For example, it is indicated in Figure 3 that at $140 \mathrm{kv}$ nearly one-third of the energy obtained in the calorimeter is due to $K$ characteristic radiation. This is considered not to be unreasonable in view of some unpublished work by the author (20) which indicated a considerable prominence of the $K$ lines from tungsten at high voltages. A rough check on the amount of the characteristic radiation may be obtained by a use of the known fact that the characteristic ray energy increases approximately with $\left(V-V_{0}\right)^{3 / 2}$ for voltages $V$ which are not large in comparison with the excitation voltage $V_{0}$. The dotted line of Figure 3 was calculated on the assumption that the intensity $I^{\prime}{ }_{K}$ of the $K$ characteristic rays obtained in the calorimeter followed the formula

$$
I^{\prime}{ }_{K}=H\left(V-V_{0}\right)^{1.3} \ldots\left(V_{0}<V<150 \mathrm{kv}\right)
$$

where $H$ is 0.085 if $V, V_{0}$, and $I^{\prime}{ }_{K}$ are in the same units as were used in formula (12). The agreement is considered very satisfactory. $^{3}$ It should be emphasized that the present methods are by no means adequate to distinguish between the exponents 1.3 and 1.5 ; the former, however, seemed to be somewhat more satisfactory in Figure 3. It may also be noted that, while $(\Delta g) K V^{3 / 2}$ gives the characteristic ray intensity in the calorimeter (where $\Delta g$ is the difference between the unbroken and the broken curves of fig. 3), no attempt is made here to obtain an expression for characteristic ray energy generated inside the anticathode, which would involve an estimation of the effective depth of production of the characteristic radiation (see Section II, 3, above).

In Figure 4 are plotted the experimental values of $g$ which would result if $I^{\prime}{ }_{c}$ were taken to be proportional to $V^{2}$, as has usually been assumed, instead of $V^{3 / 2}$. The values for efficiency previously published by the author $(21,22)$ was based on the $V^{2}$ law, and it was believed at the time that the errors necessary to account for the inconsistencies, which are, after all, not considerable for this type of work, were due, perhaps, partly to experimental error and partly to insufficiently accurate formulas for spectral intensity distribution and absorption in the anticathode. However, it is believed that in the present paper these estimates are made as satisfactorily as present experimental data will permit. It is readily seen in Figure 4 that below $69 \mathrm{kv}$ the agreement with the calculated curve is not nearly so satisfactory as in Figure 3 where $V^{3 / 2}$ was assumed, and that also in Figure 4 there is obtained no indication of an adequate amount of characteristic ray intensity above the excitation potential $V_{0}=69 \mathrm{kv}$.

More confidence in the assumed spectral energy distributions and the proportion of characteristic ray energy (and thus in the $V^{3 / 2}$ assumption) may be gained by calculating the decrease of intensity which should be observed for the addition of various thicknesses of copper filter, on which Rump obtained some measurements. The top curve of Figure 5 is identical with Figure 3 ; the other curves

3 The disagreement with the observed value for $114 \mathrm{kv}$ is probably due to a typographical error, of which several exist in Rump's paper. This is borne out by the curves plotted in Figure 5, which show good agreement with the calculations for this voltage when various filters are used. 


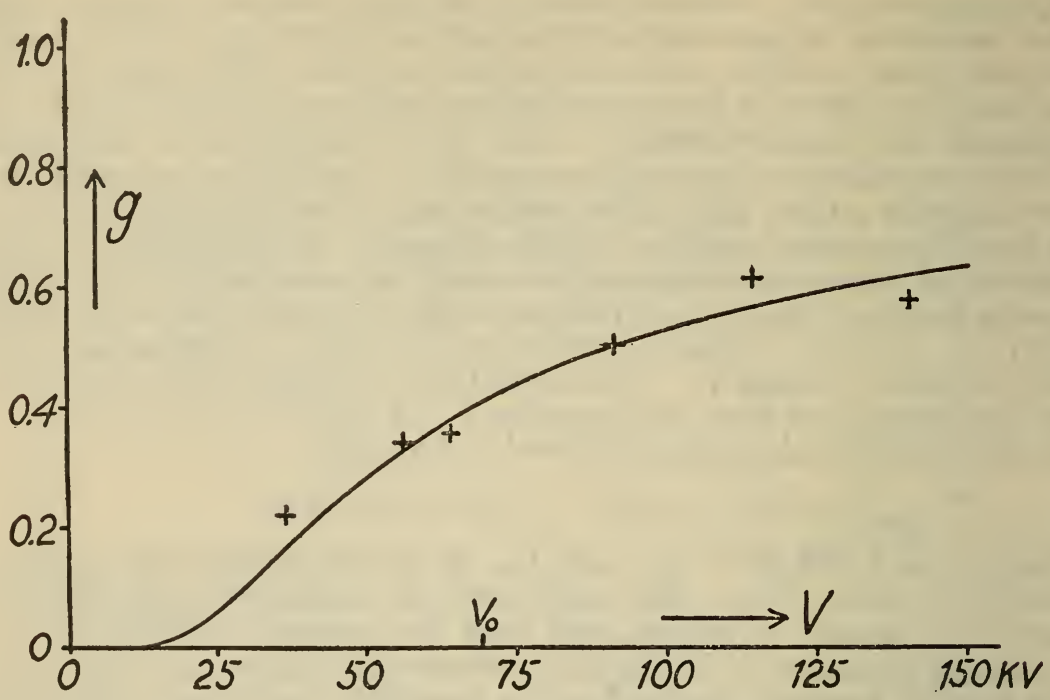

FIGURE 4.-Assuming continuous spectrum intensity proportional to $V^{3}$

Note that the agreement is not so good as in Figure 3 at low voltages, and that above the excitation potential ( $\left.V_{0}\right)$ of the $\mathrm{K}$ lines there is not adequate allowance here for characteristic ray intensity.

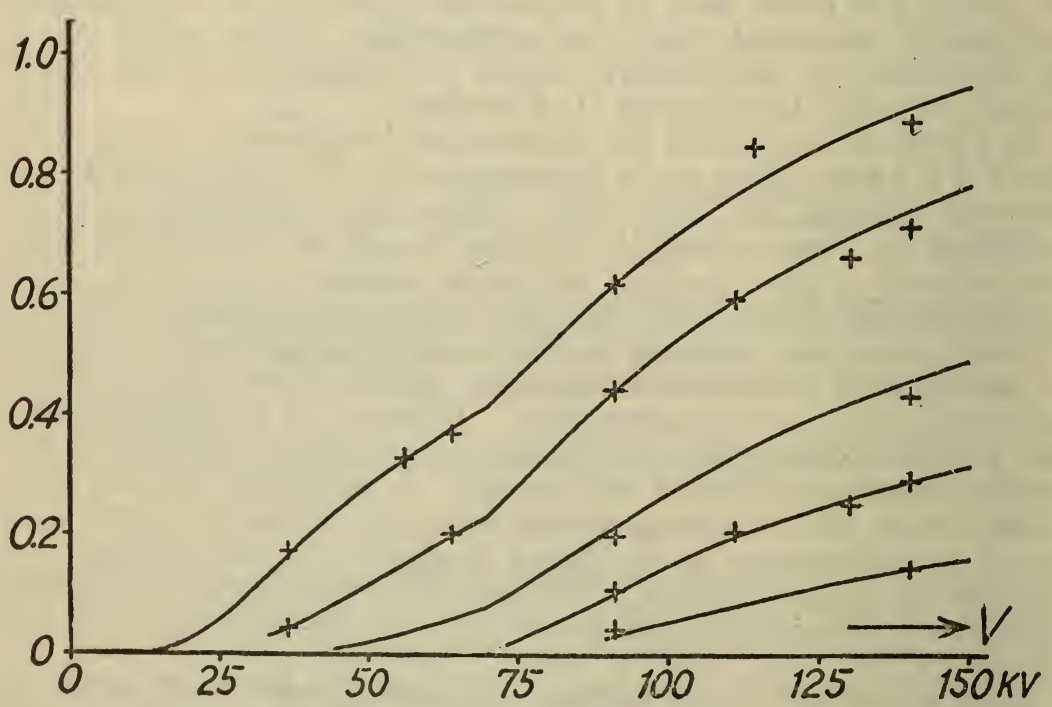

FIGURE 5.-Use of various thiclnesses of copper filter-a test of the assumed spectral intensity distributions and proportion of characteristic ray intensity 
are similar calculations, for filtrations of $0.1,0.5,1.0$, and $2.0 \mathrm{~mm}$ copper. The irregular distribution of observed points (crosses) about the calculated curves indicates that the disagreements are probably mostly due to experimental errors. However, this is not a very sensitive test, considering the experimental uncertuinties, and its value as a check on the assumptions must not be overestimated. An estimate of its value as a check may be obtained from Figure 6 , where Rump's values for the intensity at $140 \mathrm{kv}$ (effective) 4 are plotted. Curve $A$ was calculated on the assumption that the radiation at $140 \mathrm{kv}$ consisted entirely of continuous spectrum, curve $C$

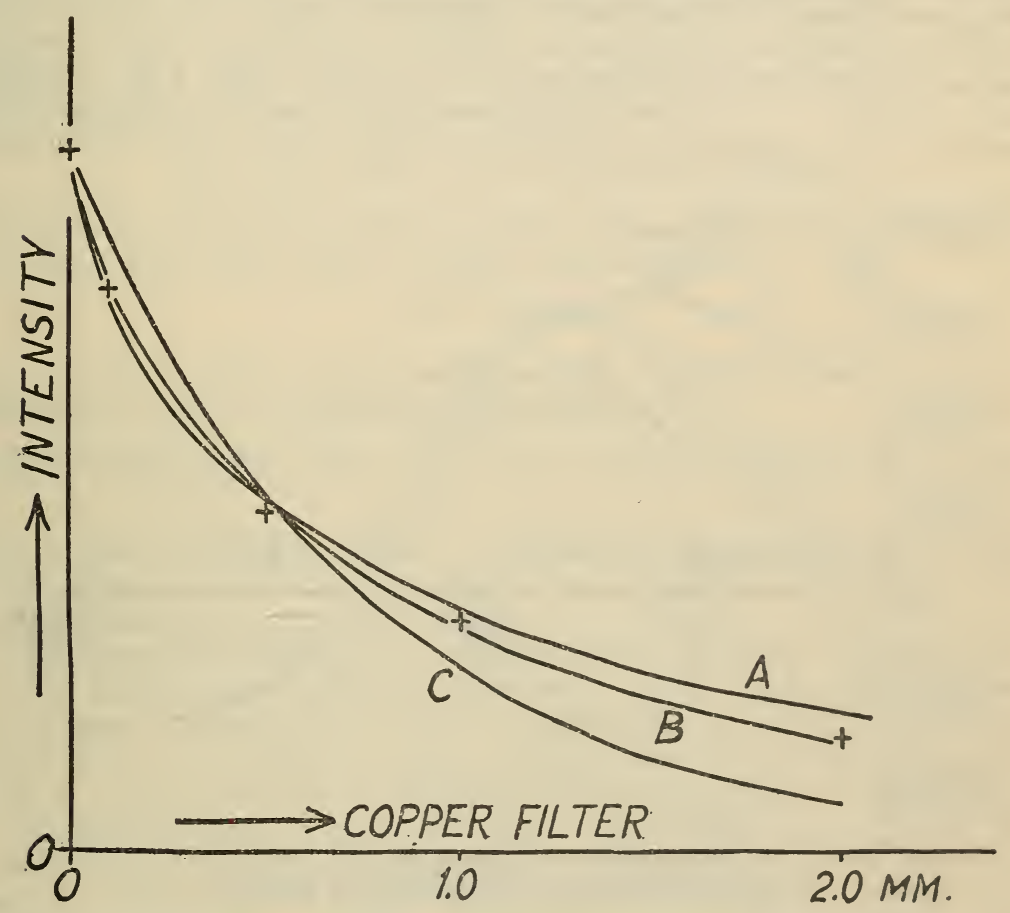

Figdre 6.-Absorption curve for the radiation at $140 \mathrm{kv}$

$A$, calculated on assumption radiation all continuous rays.

$B$, assuming radiation after absorption in anticathode and the equivalent of $1.23 \mathrm{~mm}$ aluminum

is one-third characteristic rays and two-thirds continuous. $C$, assuming radiation entirely characteristic rays.

assumed the radiation to be all $K$ characteristic rays, and curve $B$ assumed a proportion of two-thirds continuous rays and one-third characteristic, for the radiation reaching the calorimeter with no copper filter.

\section{INTENSITIES IN DIFFERENT DIRECTIONS}

In previous estimates of efficiency it has usually been assumed that the X-ray energy produced inside the anticathode is radiated equally in all directions. It is well known that this is not actually

4 Due to the limited capacity of the condensers in the flter system used by Rump, the voltage varied between limits which were about 7 per cent from the mean at $140 \mathrm{kv}$. 
the case, but on account of a lack of experimental work for the various directions, and especially the impossibility, on account of absorption in the target, of ever measuring the energy which is emitted in one hemisphere (that is, into the target), no adequate basis has been available for a better estimate. However, on account of the recent work on thin targets, a satisfactory method of making these corrections seems now to be available.

As previously pointed out (5), a thick target spectrum may be regarded roughly as being composed of two parts $(A)$ a part similar to a thin target spectrum and showing the same kind of variation with direction relative to the cathode stream, and $(B)$ a part due to completely diffused cathode rays, which, therefore, shows no variation with direction. On this basis it was shown that the variation with direction of the thick target spectrum may be described by permitting the constant $a$ of formula (11) to vary with direction.

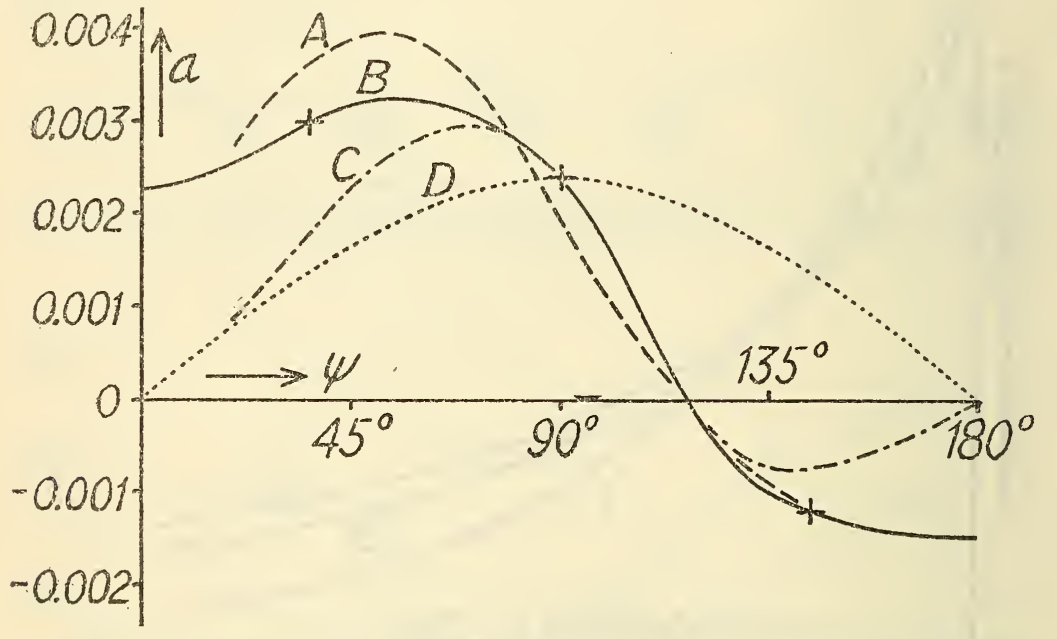

FIGURE 7.-Calculations used for correcting for variation of $X$-ray intensity with direction relative to the cathode stream

The variation of $a$ is not expected to be capable of being predicted solely from the thin target work, however, on account of the fact that part $A$ is considerably contributed to by cathode rays that are partly diffused. It is unfortunate, therefore, that data on tungsten is not available to guide the assumption about the quantitative variation of $a$. However, due to the minor importance of the correction, the variation may be guided with sufficient accuracy for present purposes by the author's work on copper (9), and a more satisfactory correction can be made directly data on tungsten become available. At $40 \mathrm{kv}$, the highest voltage at which the above-mentioned work is reliable, the values thus selected for $a$ for $\psi=36^{\circ}, 90^{\circ}$, and $144^{\circ}$ ( $\psi$ being the angle between cathode beam and measured $\mathrm{X}$ rays) were $0.0030,0.0024$, and -0.0012 , respectively; - these values are plotted in Figure 7 (crosses). Curve $B$ of Figure 7 was selected for the continuous variation of $a$ with $\psi$; this selection was partly guided by curve $A$, which is the variation of intensity with $\psi$ (but plotted here with a different zero of intensity) observed by Kulenkampff 
(23) at $38 \mathrm{kv}$ for a thin target of aluminum (it is indicated in some work by the author (5) that a similar variation holds for heavier elements). Curve $B$, being contributed to by partially diffused cathode rays, is chosen with broader maxima and minima than curve $A$. Curve $D$ is proportional to $\sin \psi$, and curve $C$ is proportional to the product of $\sin \psi$ and curve $B$. These curves will be of use below.

\section{EFFICIENCY OF X-RAY PRODUCTION}

If X-ray intensity were independent of the direction of emission, and if there were no complications due to reflection of cathode rays from the focal spot, the efficiency of production of continuous spectrum X rays would be calculable directly from formula (12) as follows: The total amount of continuous spectrum X-ray encrgy given out over the whole sphere would be

$$
\text { 4. } \pi \times 100^{2} \times 0.043 V^{3 / 2} i=5,400 V^{3 / 2} i \mathrm{ergs} / \mathrm{sec} .
$$

where $V$ is in kilovolts and $i$ is tube current in milliamperes. Using the same units, the energy supplied to the tube is

\section{$10^{7} \mathrm{~V} i \mathrm{ergs} / \mathrm{sec}$.}

The ratio of these two quantities may be termed the "apparent efficiency" for the continuous radiation; that is,

Apparent efficiency $=540 \times 10^{-6} V^{\frac{1}{2}} \ldots(Z=74) ;(40 \mathrm{kv}<V<150 \mathrm{kv})$

this is the quantity which is of importance in practical work, since it is a measure of the intensity obtainable for the experimental arrangement which is usually used.

The quantity of importance theoretically, in that it is a measure of the amount of continuous spectrum energy $\left(J_{c}\right)$ radiated in all directions in the hypothetical tube described in Section $I$, may be called the "true efficiency" $\left(E_{c}\right)$; this has been defined above as $\mathcal{J}_{c} / V i$. It is $E_{c}$ which should be compared with calculations such as those of Kramers (3) and Neunhoffer (24). To obtain $E_{c}$ two further corrections are necessary: (1) Integration of intensities for all directions, and (2) allowance for reflection of cathode rays at the target face. On account of the small magnitude of each of the corrections, the order of making them is inconsequential.

The integration for the various directions may be carried out according to the formula

$$
\int_{0}^{\pi} I^{\prime}{ }_{c}(\psi) \sin \psi d \psi
$$

where the factor $\sin \psi$ is introduced on account of the fact that $I^{\prime}{ }_{c}$ is an intensity per unit solid angle instead of a quantity of energy per unit $\Delta \psi$. Now $I^{\prime}{ }_{c}(\psi)$ may be regarded as being composed of two parts, $I^{\prime}{ }_{c 1}$ and $I_{c 2}^{\prime}$, which correspond to the two terms on the righthand side of formula (11); that is

$$
\begin{aligned}
I^{\prime}{ }_{c 1} & =C Z\left(\nu_{0}-\nu\right) \\
I^{\prime}{ }_{c 2} & =C Z^{2} \nu_{0}\left(1-e^{-16\left(\nu_{0}-\nu\right) / \nu}\right) u(\psi) \\
11295^{\circ}-30-6 &
\end{aligned}
$$


(Primed quantities are used in order to be consistent with the definitions in Section II, in which energies associated with the focal spot were denoted by primed quantities.) According to Section V, $I^{\prime}{ }_{c 1}$ does not vary with $\psi$, but $I^{\prime}{ }_{c 2}$ does on account of the variation of $a$ with $\psi$. Accordingly, the integral (15) becomes

$$
I^{\prime} \int_{01}^{\pi} \sin \psi d \psi+\int_{0}^{\pi} I_{c 2}^{\prime} \sin \psi d \psi=2 I^{\prime}{ }_{c 1}+\int_{0}^{\pi} I^{\prime}{ }_{c 2} \sin \psi d \psi
$$

Now the calculation of the apparent efficiency (formula (14)) was really equivalent to integrating equation (16) with a constant value of $I^{\prime}{ }_{c 2}^{2}$ equal to the value $\left(I^{\prime}{ }_{c 2}\right)_{90^{\circ}}$ observed for this quantity at $\psi=90^{\circ}$; the integral would then turn out to be

$$
2 I^{\prime}{ }_{c 1}+2\left(I^{\prime}{ }_{c 2}\right)_{90^{\circ}}
$$

and the factor by which the apparent efficiency must be corrected to allow for the variation of intensity with $\psi$ is the ratio (16) to (17). In evaluating this ratio, it may be noted that $I^{\prime}{ }_{c 1} /\left(I^{\prime}{ }_{c 2}\right)_{90^{\circ}}$ may be obtained from an integration of formula (11), and that $2\left(I_{c 2}^{\prime}\right)_{90^{\circ}}$ $\int_{0}^{\pi} I^{\prime}{ }^{\prime} \sin \psi d \psi$ is the ratio of areas under curves $D$ and $C$ of Figure

7. It turns out that the correction factor for $40 \mathrm{kv}$ is 0.91 .

Finally we consider the correction for reflection of cathode rays from the target face. Unfortunately, this effect has been only incompletely investigated, and apparently the best that can be done as a correction for the effect is to suppose, as Rump did, that 20 per cent of the total X-ray energy becomes "off-focus" radiation. Coolidge (7) has estimated the amount of radiation coming from the back of the target to be about one-ninth of the radiation from the focal spot, and allowing for both front and back of target and stem, this seems a reasonable correction. Thus we find the efficiency $E_{c}$ of production of continuous spectrum $\mathrm{X}$ rays in tungsten at $40 \mathrm{kv}$ to be

$E_{c}=0.91 \times 1.25 \times 540 \times 10^{-6} \times 40^{\frac{1}{2}}=0.0039$. $(\mathrm{V}=40 \mathrm{kv}) ;(Z=74)$.

On account of a lack of knowledge of the variation of intensity with $\psi$ for voltages higher than $40 \mathrm{kv}$, not much can be said at present about the value of $E_{c}$ for other voltages. However, on account of the increasing concentration of intensity in the forward angles (values of $\psi$ less than $90^{\circ}$ ) for higher voltages, the total continuous spectrum energy $J_{c}$ may be expected to increase somewhat faster than $V^{3 / 2}$. Supposing it to increase proportional to $V^{2}$, making the efficiency proportional to $V$, and assuming $E_{c}$ proportional to $Z$ (as is usually accepted), we should have for the efficiency, using the value just found at $40 \mathrm{kv}$ to determine the constant,

$$
E_{c}=13 \times 10^{-7} Z V
$$

where $V$ is in kilovolts. Rump's value (1) for the constant in this formula was about $15 \times 10^{-7}$; Kulenkampff's values (2) were $(9.5 \pm 3) \times 10^{-7}$, based on his own work with resolved radiation at 7 to $12 \mathrm{kv}$, and $(8 \pm 2) \times 10^{-7}$, based on other work with unresolved radiation; Kramers (3) obtained $9.2 \times 10^{-7}$ from a theoretical calculation of the radiation from electrons in hyperbolic orbits. 


\section{EFFICIENCY AND THE THOMSON-WHIDDINGTON CONSTAN'T}

It appears not to be generally appreciated that the X-ray energy generated by cathode rays in penetrating matter is dependent on the value of the constant $b$ in the Thomson-Whiddington-Bohr law (equation (3)). This fact follows at once from an analysis by Webster (25), and may also be shown as follows: According to current theory, a cathode ray can generate an $\mathrm{X}$ ray only when it has a close encounter with an atomic nucleus; that is, when the path of the cathode ray as it approaches an atom is such that an extension of this path lies within a certain small area $\tau$ surrounding the nucleus. 'The probability that a cathode ray will have one of these close encounters in a path length $d x$ is

$$
\tau N d x
$$

where $N$ is the number of atoms per unit volume. Or, utilizing equation (3) above, the probability of a close encounter while the cathode ray is losing an amount of speed corresponding to $d V$ (kilovolts) is

$$
\frac{2 \tau N V d V}{b}
$$

Thus, if the constant $b$ could be made smaller for an X-ray anticathode (strictly $b / N$ must be made smaller) the cathode rays would penetrate a great number of atoms before being slowed by a given amount, the total number of close encounters with nuclei would be increased, and the number of X-ray quanta generated would be greater. Such a change of $b / N$ (but in any case probably only a very small change) may be readily imagined to be associated with a change of crystal structure for example.

The above conception has some interesting applications. It is generally accepted that the intensity of continuous spectrum $\mathrm{X}$ rays obtained from various anticathodes increases regularly with the atomic number $(Z)$ of the material of the anticathode, being approximately proportional to $Z$. Ulrey's experimental results (26), however, indicated that the intensity did not increase regularly, but showed small periodic fluctuations correlating with the periodic variation of chemical properties; these fluctuations have often been thought to be due to some kind of experimental error. If the production of $\mathrm{X}$-ray energy were simply dependent on the collisions between cathode rays and nuclei, there would, indeed, be no basis for assuming any periodicity here, since the electrical properties of the region immediately surrounding the nucleus of atoms should increase in a regular manner with atomic number. However, since the $\mathrm{X}$-ray energy generated is indirectly influenced by $b / N$, any atomic property which influences the magnitude of $b / N$ may also influence the $\mathrm{X}$-ray energy. Now $b / N$ is a measure of the energy lost by the cathode rays in passing through the material; this energy is used chiefly to ionize and excite the outer energy levels of the atoms, and depends to a considerable extent not only on the number of loosely bound electrons in the atoms, but also on their energy of binding. As is well linown, this energy of binding does not increase regularly with atomic number, but shows a periodic variation coinciding with the periodic variation of chemical 
properties; for example, the valence electrons of the alkalies and alkaline earths are much less firmly bound than the outer electrons of the halogens. Thus a small periodic variation with atomic number, such as was observed by UTrey, may be expected for the X-ray intensity obtained from various anticathodes. It is interesting in this connection that a comparison (2) of Ulrey's with Kaye's (27) results indicates that the two pieces of work, which were done under entirely different conditions are in agreement as to the position and magnitude of these variations.

It may be remarked incidentally that, as is obvious from the above discussion, a measurement of the X-ray intensity from a material may be used for an indirect determination of the Thomson-Whiddington constant $b$. Now the value of $b$ has been determined by direct methods for only a very few elements (on account of the requirement that the element be capable of being rolled or beaten into very thin foils) and these have of necessity been in only certain chemical groups. However, the X-ray emission has been measured from a comparatively large number of elements, covering other chemical groups. The fact that the X-ray emission from all these elements has shown only the minor irregularities noted above is adequate evidence that $b / N$ will show, with varying atomic number, only corresponding minor fluctuations. This was the basis, in Section II, 3 , above for not questioning the application to molybdenum and tungsten of Terrill's formula ${ }^{5}$ for $b$, although $b$ has not yet been observed directly for these elements.

The relation between X-ray intensities and the quantity $b$ also has application to the anomaly mentioned above (Sec. II, 3) with regard to the observations of Webster and Hennings on the effective depth of production $(\bar{x})$ of continuous spectrum $\mathrm{X}$ rays. Their method depends on the measurement of the discontinuity in the continuous spectrum from an anticathode, the discontinuity being observed at a critical absorption frequency $\left(\nu_{a}\right)$ of the target material. As they pointed out, in order to deduce $\bar{x}$, it must be assumed that the continuous spectrum as emitted has no discontinuity at $v_{a}$; that is, that the entire observed discontinuity must be due to the absorption of the rays in their path through the target material. Now there are several conceivable ways of producing just such a discontinuity prior to the absorption along the path: One possibility is a process (somewhat similar to Auger's compound photoelectric effect (28) with fluorescent rays) in which the continuous spectrum quanta may have an especially high probability of being absorbed in the atoms within which they are produced. Another possibility is that $b$ may undergo a sudden change for cathode ray speeds which will just excite frequency $\nu_{a}$; Schonland (29) has speculated on the possibility of such a change, and has searched for it experimentally, but under such conditions as would probably not have allowed the detection of so small a change as is necessary to explain the present anomaly. The reason for renewing the discussion of the point at the present time is, of course, to justify the use of the formula for $\bar{x}$ proposed above. However, it seems also that such a change in $b$ would have some very fundamental

The ahove considerations are entirely apart from the fact that Terrill's formula must itself introduce into $b$ some small irregularities which are due to the fact that atomic weight is not a perfectly regular function of atomlc number. From a theoretical standpoint, a more satisfactory expression would be $b=f(\rho Z / A)$ where $A$ is the atomic weight of the material, and $f$ is a function to be determined; this causes $b$ to be dependent ou atomic number instead of atomic weight. It turns out, however, that $b$ is closely proportional to $\rho$, and rather than use a form for $f$ which is less simple than the liner form, the formula $b=40 \times 10^{4} \rho$ is generally
glven. 
theoretical consequences, and experiments designed to test the point are now in progress in the author's laboratory.

\section{DISCUSSION}

Probably the greatest degree of uncertainty in the above calculations is the 20 per cent correction for stray cathode rays; this estimate was made by a photographic method, and not much is known about its variation with tube voltage. However, it should be pointed out, that this concerns only the calculation of the "true," or theoretical efficiency $E_{c}$; the equations of practical importance $(11,12,13$, and 2$)$, in that they describe the intensity $I^{\prime}$ actually obtainable from the focal spot, are independent of the 20 per cent correction; and of course, when better measurements on the stray energy are available, they can be applied directly to the present work in order to obtain a better estimate of $E_{c}$.

Another uncertainty which affects only $E_{c}$ and not $I^{\prime}$ is in the correction for the intensity obtained in various directions. A serious estimate of the adequacy of the present correction for this effect can not be made until work is available for a target of atomic number in the vicinity of tungsten, but it is scarcely conceivable that the error in the present work due to this cause can be over a few per cent. It may be pointed out that whatever uncertainty now exists with regard to this correction is due simply to a lack of experimental data, and is not due to the fact that intensities radiated into the massive target can never be measured.

An uncertainty which applies to both $E_{c}$ and $I^{\prime}$ is due to the fact that the voltages used by Rump were not so steady as could be desired, but varied about $10 \mathrm{kv}$ from the mean. It is difficult to estimate the possible errors introduced by this variation, on account of the fact that the voltage wave form of a circuit of the type used by Rump is not a simple one. Supposing a sinusoidal variation, however, it is readily shown ${ }^{6}$ that at $40 \mathrm{kv}$, for example, the correction would bo only about 5 per cent; at higher voltages the correction is even less serious. If the observed points below $V_{o}$ on the curve of Figure 3 were corrected on this basis, the observations would more closely parallel the calculated curve than they do at present. This small correction has, as a matter of fact, already been applied to the numerical results above involving low voltages (equations (12), (14), (18)), and the value of $E_{c}$ for $40 \mathrm{kv}$.

6 At $40 \mathrm{kv} g$ (fig. 3) is roughly proportional to $V^{2} / 2$, making $q I^{\prime}$ proportional to $V^{3}$. A lso, at this voltage, the variation from the mean voltage was about $7 \mathrm{kv}$. Therefore the variation of voltage may be described by

$$
V=40+7 \sin y
$$

where $y$. is a variable increasing linearly withytime. The intensity actually obtained inside the calorimeter is

$$
P \int_{0}^{2 \pi}(40+7 \sin y)^{2} d y
$$

where $P$ is a constant, and the intensity which would have been obtained if the voltage had been steruly at $40 \mathrm{kv}$ is

$$
P \int_{0}^{2 \pi}(40)^{3} d y
$$

Thus the correction factor by which an observed point (fig. 3) at $40 \mathrm{kv}$ should he multiplied is the ratio of (20) to (19), which turns out to be 0.954 . 
The other uncertainties, associated with correction for absorption in the anticathode, and spectral intensity distribution, are probably comparatively minor; the formulas set up for these corrections are believed to be quite satisfactory from the standpoint of present experimental data. ${ }^{7}$ Moreover these formulas are of sufficient generality to be of use in future work on X-ray efficiency, at least until better experimental data is available for making these difficult corrections.

\section{SUMMARY}

1. The physical conditions inside gas filled tubes are discussed, and it is concluded that, for definite interpretation of results, hot cathode tubes should be used for experimental estimates of X-ray efficiency.

2. A general formula (formula (2)) for absorption of continuous spectrum radiation in the anticathode is set up and compared with available experimental data.

3. A general formula (formula (11)) is given which describes the spectral intensity distribution in the general radiation emitted at an angle $(\psi)$ of $90^{\circ}$ from the direction of the cathode stream.

4. Calculations based on some experimental work by Rump lead to the conclusion that, between 40 and $150 \mathrm{kv}$. and for $\psi=90^{\circ}$, the unresolved intensity in the general radiation generated inside a tungsten anticathode is proportional to the $3 / 2$ power of the voltage $(V)$ instead of to $V^{2}$ as has usually been assumed (formula (12)).

5. A basis for estimating absolute intensities and spectral intensity distributions for any value of $\psi$ is provided (fig. 7 ).

6 . The intensity of the $K$ characteristic radiation obtainable from tungsten at voltages up to $150 \mathrm{kv}$ is described quantitatively (formula (13)).

7. Formulas (2), (11), (12), and (13), together with Figure 7 provide a means for describing quantitatively the absolute intensity and spectral intensity distribution (including in the case of tungsten the proportion of $K$ characteristic rays) of the continuous radiation for any direction $(\psi)$ obtainable from an X-ray tube under a wide range of practical conditions.

8. The efficiency $E_{c}$ of production of continuous spectrum $\mathrm{X}$ rays inside a tungsten anticathode is estimated for $40 \mathrm{kv}$. It is pointed out that there is not yet adequate basis for extending the estimate of $E_{c}$ over a wide range of voltage.

9. The bearing of the Thomson-Whiddington constant on the generation of X-ray energy is pointed out, and its application made to the explanation of some results by Ulrey which indicated that X-ray efficiency showed a periodic variation with atomic number.

The following tabulation may be of interest for purposes of illustration and numerical comparisons:

7 There remains an apparent minor difficulty in this connection: Formula (11) (spectral intensity distri bution) was derived $(13,9)$ from data of the isochromat type assuming the $V^{2}$ law for unresolved intensity. Consequently, a discrepancy appears when a $V^{3 / 2}$ law is found above (formula (12)). However, it may be recalled that in the derivation of formula (11) an approximation was made (neglect of a term corresponding to the exponential term of formula (11)) which was in the direction of assuming $V^{3 / 2}$ instead of $V^{2}$. This, together with the consideration that any remaining differences would probably not be detectable experimentally, removes the apparent discrepancy. 
Tungsten anticathode; Constant potential; 1 milliampere tube current

\begin{tabular}{|c|c|c|c|c|}
\hline & $40 \mathrm{kV}$ & $69 \mathrm{kV}$ & $100 \mathrm{kv}$ & $150 \mathrm{kv}$ \\
\hline $\begin{array}{l}\text { Total continuous spectrum energy produced inside anticathode, allowing } \\
20 \text { per cent for cathode-ray reflection losses. (ergs/sec.) }\end{array}$ & \multirow[t]{2}{*}{$\begin{array}{r}1.23 \\
\times 10^{6}\end{array}$} & \multirow[t]{2}{*}{$\begin{array}{r}3.66 \\
\times 10^{6}\end{array}$} & $\begin{array}{r}7.70 \\
\times 10^{6}\end{array}$ & \multirow[t]{2}{*}{$\begin{array}{r}17.3 \\
\times 10^{6}\end{array}$} \\
\hline $\begin{array}{l}\text { Continuous spectrum intensity which would have been obtained out- } \\
\text { side of tube in the usual } 90^{\circ} \text { position if all absorptions (including that } \\
\text { in anticathode) had been negligible. (ergs } / \mathrm{sec} . \mathrm{cm}^{2} \text { at } 1 \mathrm{~m} \text { from focal }\end{array}$ & & & & \\
\hline $\begin{array}{l}\text { Spot } \\
\text { Continuous spectrum intensity actually obtained outside tube for }\end{array}$ & 10.9 & 24.6 & 43.0 & 79.1 \\
\hline $\begin{array}{l}\text { which the walls and other filtration (except anticathode) are equiva- } \\
\text { lent to } 1.23 \mathrm{~mm} \text { aluminum. (ergs } / \mathrm{sec} \text {. } \mathrm{cm}^{2} \text { at } 1 \mathrm{~m} \text { from focal spot) }\end{array}$ & 2.18 & 10.0 & 22.5 & 50.0 \\
\hline $\begin{array}{l}\text { Intensity in } K \text { characteristic radiation actually obtained outside above } \\
\text { tube (same units) }\end{array}$ & 0 & 0 & 7.3 & 24. 2 \\
\hline
\end{tabular}

\section{REFERENCES}

1. W. Rump, Zeits. für Physik, 43, p. 254; 1927.

2. H. Kulenkampfi, Handbuch der Physik, 23.

3. H. A. Kramers, Phil. Mag., 46, p. 869; 1923.

4. D. L. Webster, P. N. A. S., 14, p. 339; 1928.

5. W. W. Nicholas, B. S. Jour. Research, 2, p. 837; 1929.

6. W. Bothe, Handbuch der Physik, 24, p. 1.

7. W. D. Coolidge, Am. J. Roentgen, 1, p. 115; 1914.

8. Ledoux-Lebard and Dauvillier, Physique des Rayons X, p. 45; 1921.

9. W. W. Nicholas, Phys. Rev., 29, p. 619; 1927.

10. E. Lorenz, P. N. A. S., 14, p. 582; 1928.

11. D. L. Webster and A. E. Hennings, Phys. Rev., 21, p. 301; 1923.

12. B. F. J. Schonland, Roy. Soc. Proc., 104, p. 235; 1923; 108, p. 187; 1925.

13. D. L. Webster and A. E. Hennings, Phys. Rev., 21, p. 312; 1923.

14. D. L. Webster, P. N. A. S., 14, p. $330 ; 1928$.

15. W. P. Davey, J. Frank. Inst., 1\%1, p. 277; 1911.

16. L. G. Davey, Phys. Rev., 4, p. $217 ; 1914$.

17. W. R. Ham, Phys. Rev., 30, p. 96; 1910.

18. H. Kulenkampfi, Ann. der Physik, 69, p. 548; 1922.

19. D. L. Webster, P. N. A. S., 5, p. $163 ; 1919$.

20. W. W. Nicholas, "Spectrum of X-Rays used in Deep Therapy," M. A. thesis, Stanford University.

21. International Critical Tables, 6.

22. W. W. Nicholas, Phys. Rev., 35, p. 128; 1930.

23. Kulenkampff, Ann. der Physik, 8\%, p. 597; 1928.

24. M. Neunhoffer, Ann. der Physik, 81, p. 493; 1926.

25. D. L. Webster, Phys. Rev., 9, p. $220 ; 1917$.

26. C. T. Ulrey, Phys. Rev., 11, p. 401 ; 1918.

27. G. W. C. Kaye, Phil. Trans. A., 209, p. 237; 1908.

28. P. Auger, J. de Phys. et Radium, 6, p. 205; 1925.

29. B. F. J. Schonland, Proc. Roy. Soc. A., 108, p. 187; 1925.

Washington, February 21, 1930. 